\title{
Bajar la presión ocular reduce el riesgo de complicaciones en pacientes con hipertensión ocular o glaucoma manifiesto
}

Maier P y col. BMJ 2005;331:134.

\section{Objetivo}

Revisar sistemáticamente la literatura sobre la efectividad del tratamiento de la hipertensión ocular (presión ocular elevada sin cambios en el disco óptico y/o campo visual) y del glaucoma primario de ángulo abierto (presión ocular elevada o normal, con cambios en el disco óptico y/o campo visual).

\section{Fuentes de Datos}

a) bases de datos: Cochrane Central Register of Controlled Trials (2004), Medline (1966-2004) y Embase (1974-2004); b) otras bases de datos de guías y reportes de evaluación de tecnologías; c) referencias de artículos relevantes; d) Se preguntó a investigadores y expertos sobre ensayos en realización.

\section{Selección de Estudios}

Ensayos controlados aleatorizados sobre tratamiento hipotensor con un grupo control concurrente no tratado y puntos de finalización apropiados (defectos de campo visual o cambios glaucomatosos en el disco óptico).

\section{Extracción de Datos}

Los autores realizaron un meta-análisis de cinco estudios sobre hipertensión ocular y dos sobre glaucoma primario de ángulo abierto (por separado); y un análisis predefinido de subgrupos de glaucoma de tensión normal, glaucoma de presión elevada y pacientes con cambios glaucomatosos inequívocos en el campo visual o disco óptico.

Extrajeron la información al momento del deterioro del campo vi- sual y de los cambios del disco óptico definitivos compatibles con glaucoma, realizando un meta-análisis del tiempo al evento entre los grupos intervención y control.

\section{Resultados Principales}

El meta-análisis de los resultados de los estudios incluidos para estimar la eficacia de bajar la presión en la hipertensión ocular mostró un efecto beneficioso del tratamiento ("hazard ratio"* 0,56 ; IC95\%: 0,39-0,81) al igual que, en el glaucoma manifiesto al bajar la presión para prevenir la progresión del glaucoma ("hazard ratio" 0,65; IC95\%: 0,49-0,87). No se observó heterogeneidad.

El número de pacientes que es necesario tratar durante cinco años para prevenir el primer defecto de campo visual o cambio en el disco definitivo glaucomatoso fue 12 (IC95\% 9-29) y para prevenir la progresión a glaucoma en un paciente, 7 (IC 95\%: 4-20). Al analizarse los subgrupos de glaucoma con presión ocular elevada y de presión normal, no se encontró certeza en cuanto al beneficio del tratamiento hipotensor en el segundo grupo.

\section{Conclusiones}

Los autores concluyen que: 1) la prevención primaria de los defectos de campo visual en pacientes con hipertensión ocular utilizando hipotensores tópicos parece ser efectiva; 2) reducir la presión intraocular en pacientes con glaucoma de ángulo abierto lleva a un retraso significativo de pérdida de campo visual, especialmente en aquellos con presión intraocular elevada.

Fuente de financiamiento: ninguna

\section{Comentario}

El glaucoma primario de ángulo abierto, por definición "neuropatía óptica multifactorial con pérdida característica de células ganglionares retinales y atrofia del nervio óptico", es la segunda causa de ceguera en Estados Unidos. Entre los múltiples factores que se asocian a su prevalencia, los más importantes son el nivel de presión intraocular (obsérvese que la presión no se encuentra en muchos textos en la definición de la enfermedad) la raza, el espesor centrocorneal, la edad y los antecedentes familiares.

Por lo menos dos estudios demostraron que la prevalencia del glaucoma primario de ángulo abierto aumenta al elevarse la presión ocular. A pesar de esta relación, existe gran variabilidad entre los individuos en la susceptibilidad del nervio óptico al daño producido por la presión intraocular. Sólo uno de cada diez pacientes con hipertensión ocular desarrollan daño de campo visual. Además de la presión, otros factores (aporte sanguíneo al nervio óptico, sustancias tóxicas para el nervio óptico y la retina, metabolismo axonal o neuronal) influirían en el progreso de la neruropatía óptica glaucomatosa. Pero dado que la presión intraocular es una causa tratable del daño al nervio óptico, es esperable que al bajar la presión intraocular se retrase el progreso de la neuropatía óptica.

Si bien en Argentina se ha seguido en las últimas décadas la fuerte influencia de la escuela del Prof. Roberto Sampaolesi, quien desde los años sesenta insistió en la importancia de bajar la presión para prevenir el progreso del glaucoma, en otros países, por ejemplo en los Estados Unidos, hasta hace poco años se discutía el rol de la presión ocular como causa del glaucoma, por lo que los hallazgos de estos estudios reforzarán la idea de intervenir sobre este factor tratable, por el momento el único, para disminuir el progreso de la enfermedad.

Como tema a analizar, sería interesante conocer la diferencia en la evolución entre aquellos pacientes tratados antes de que desarrollen daño, comparados con aquellos en quienes se espera a la aparición del primer defecto de nervio óptico o campimétrico glaucomatoso, ya que esto permitiría conocer el tiempo que muchos pacientes podrían estar sin tratamiento y sin daño.

\section{Conclusiones del comentador}

La presión ocular es, por el momento, el único factor asociado al desarrollo de glaucoma que cuenta con evidencia que respalda su tratamiento con el objetivo de disminuir la progresión de la enfermedad. 